Original Research Article

\title{
Effect of agomelatine on psychomotor function tests in healthy human volunteers
}

\author{
G. Sirisha ${ }^{1 *}$, Shovan $\mathbf{P}^{2}$
}

${ }^{1}$ Department of Pharmacology, Kamineni Institute of medical Sciences, Narketpally, Telangana, India ${ }^{2}$ Department of Clinical Pharmacology, Nizam's Institute of Medical Sciences, Hyderabad, Telangana, India

Received: 17 October 2017 Accepted: 24 November 2017

\section{*Correspondence to:}

Dr. G. Sirisha,

Email: dr.sireesha.g@gmail.com

Copyright: (C) the author(s), publisher and licensee Medip Academy. This is an openaccess article distributed under the terms of the Creative Commons Attribution NonCommercial License, which permits unrestricted noncommercial use, distribution, and reproduction in any medium, provided the original work is properly cited.

\begin{abstract}
Background: Agomelatine is a melatonergic agonist that acts specifically on MT1/MT2 melatonergic receptors and 5-HT2C antagonism. The present study was taken up to evaluate the effect of Agomelatine $25 \mathrm{mg}$ on psychomotor function in healthy human volunteers.

Methods: The effect of Agomelatine was studied in 12 healthy volunteers of either gender. The study was a randomised, cross over, placebo controlled study, done after obtaining permission from NIMS Institutional Ethics Committee and informed consent taken from all the subjects, after briefly explaining the study procedure and training them adequately. Psychomotor function was assessed using Choice reaction time (CRT), Critical Flicker fusion test (CFFT), Digit letter substitution test (DLST), Six letter cancellation test (SLCT), Card sorting test (CST) and Visual analog scale (VAS). Psychomotor function tests were performed, 90 minutes after administering Agomelatine $25 \mathrm{mg}$ or placebo. Washout period of seven days was allowed between the cross over. Statistical analysis was done by comparing groups using unpaired $t$ test.

Results: There was significant decrease in the mean percentage of time $(p<0.01)$ in CRT in Agomelatine group $(20.09 \pm 9.47 \%)$ when compared to placebo $(10.48 \pm 3.68 \%)$. Improved mean percentage of performance was seen in CFFT with Agomelatine $(6.2 \pm 2.1 \%)$ compared to placebo $(9.11 \pm 2.99 \%)$. No significant difference was noted in the performance of DLST, SLCT and CST. Drug was subjectively rated as sedative on VAS.

Conclusions: There is no significant effect of Agomelatine on psychomotor performance. CNS processing of information also improved. Agomelatine can thus be safely administered to depressed patients.
\end{abstract}

Keywords: Agomelatine, Information processing, Psychomotor performance, sedative, Visual analog scale

\section{INTRODUCTION}

Depression is a family of complex multifactorial illnesses that are characterized by disruptions of several physiological, neuroendocrine, and behavioral processes. ${ }^{1}$ According to a WHO report, the depressive disorders account for the fourth leading cause of global burden of disease and by the year 2020 they are expected to be the second highest cause of morbidity. Circadian rhythm abnormalities, as shown by sleep/wake cycle disturbances, constitute one the most prevalent signs of depression; advances or delays in the circadian phase are documented in patients with major depressive disorder (MDD), bipolar disorder or seasonal affective disorder (SAD)., ${ }^{2,3}$ Disturbance of the sleep/wake cycle is one of the diagnostic criteria for depression, and people with major depression commonly experience changes in sleep/ wake cycle regulation that are seen as abnormal total sleep duration, poor sleep efficiency, overwhelming rapid eye movement (REM) sleep, and early morning awakening.

Melatonin has been shown to improve sleep and is involved in the regulation of the sleep-wake cycle. 
Agomelatine is a melatonergic agonist acting on $\mathrm{MT}_{1} / \mathrm{MT}_{2}$ melatonergic receptors and simultaneously exhibits 5$\mathrm{HT}_{2 \mathrm{C}}$ antagonism, a property which is utilized by current antidepressants. ${ }^{2,3}$

Agomelatine has no effect on monoamine uptake nor has affinity for other receptors. Therefore, as compared to other antidepressants, it has less gastrointestinal side effects and sexual dysfunction. It has no cardiovascular side effects as reported in clinical trials which were earlier performed. $^{2}$

Agomelatine is a novel atypical antidepressant having unique pharmacological property that regulates the circadian rhythm. Its benign tolerability profile makes it an effective alternative for patients who are refractory and intolerant to currently available antidepressant drugs. ${ }^{4}$

Psychomotor performance results from coordination of sensory and motor systems through integrative and organizational process in brain. ${ }^{5}$ The processing of sensory information is influenced by personality, memory and individual motivation. Sensory, motor and central coordination of psychomotor performance can be evaluated by standard validated battery of psychomotor function tests (automated or manual).

Psychomotor retardation or agitation is a cardinal sign in depression. In patients with melancholic features, psychomotor dysfunction is a key symptom for depressed patients. ${ }^{6}$ The optimum profile of an antidepressant should include no detrimental effect on cognitive and psychomotor functions. The present study was thus conducted to evaluate the effect of tablet Agomelatine $25 \mathrm{mg}$ single oral dose on psychomotor functions in healthy adult volunteers.

\section{METHODS}

The present study was a prospective, randomized, doubleblind placebo controlled, cross over study conducted in the Department of Clinical Pharmacology and Therapeutics, Nizam's Institute of Medical Sciences, Hyderabad, Telangana, India. Prior to the study, approval from Institutional ethics committee was taken and written informed consent was taken from the study subjects. Twelve healthy adult volunteers aged between 18 to 25 years, with BMI of 18.5 to $24.9 \mathrm{~kg} / \mathrm{m}^{2}$ were enrolled in the study. The study was done in a period of two months (January-February 2015). Subjects who were able to comprehend and perform the tests and atleast completed their primary education were included in the study. Exclusion criteria included those with hypertension, hyperglycemia, impaired hepatic or renal function, and cardiac arrhythmia, history of smoking, alcoholism, malignancy or stroke, on benzodiazepines and neuroleptics abuse, any disease requiring active treatment or with herbal supplements. Subjects having history of hypersensitivity, mental illness, history of occupation with machinery or driving, those who were unwilling to participate were also excluded. All subjects were screened by taking a detailed history, clinical examination and laboratory tests. Training on psychomotor tests was given to all subjects on two different occasions prior to the study. The trial was registered in CTRI (CTRI/2017/06/008793).

After screening, all the eligible subjects were enrolled in the study. Compliance to overnight fasting, good sleep and abstaining from alcohol (at least 48 hours), smoking, caffeine containing beverages (10 hours) was ensured. Subjects were randomised to receive either tablet agomelatine $25 \mathrm{mg}$ single oral dose or a matching placebo. A wash out period of seven days was maintained between the two study periods.

Battery of tests were conducted to assess psychomotor function of the subject which also included VAS for sedation. Tablet Agomelatine $25 \mathrm{mg}$ or identical placebo was administered along with $240 \mathrm{ml}$ of drinking water, under direct supervision on fasting condition. Then the subjects were allowed to relax. The same battery of tests, VAS was repeated after 90 minutes of drug administration. Standard breakfast and lunch were served at three and six hours of drug administration. Subjects were continuously monitored for adverse drug reactions throughout the study and till discharge 12 hours after drug administration.

The battery of psychomotor function test consisted of the following:

\section{Choice reaction time $(C R T)^{7}$}

The time taken by the subject to respond the selected colour (red or green) and lift his finger from corresponding button, after receiving an auditory cue is taken as choice reaction time. Mean of 3 readings is taken.

\section{Critical flicker fusion test $(\text { CFFT })^{8}$}

This is done using Critical flicker fusion apparatus. The subject is asked to look at the flickering light and frequency is increased constantly till he observes that flickering light forms constant fused light, which is considered as flicker to fusion frequency. The frequency of light is then adjusted to maximum frequency, and is consistently decreased to frequency at which when the fused light begins to flicker which is recorded as fusion to flicker frequency.

This flicker to fusion and fusion to flicker is regarded as one cycle. This is repeated for 3 cycles at an interval of 5 minutes between each and mean will be considered. Background field intensity, size of image, surrounding intensity, viewing distance were kept constant throughout the test.

\section{Six letter cancellation test $(\text { SLCT })^{9}$}

Subject was instructed to slash off printed alphabets according to the key in 90 seconds. The total number of 
alphabets slashed were recorded. This test was done for 3 times with an interval of 5 minutes between each and the mean was recorded.

\section{Digit letter substitution test $(\text { DLST })^{9}$}

Subject was instructed to write the corresponding alphabet in boxes provided below the numbers in rows. The subject was instructed to complete as many substitutions as possible in 90 seconds. Total no. of correct substitutions and total no. substitutions attempted was considered. This test was done 3 times at an interval of 5 min and mean was calculated.

\section{Card sorting test (CST)}

Subject was instructed to sort out a pack of 52 cards kept in random fashion into four groups by their symbols. The total time taken for sorting will be considered as card sorting time. Three readings were taken with a gap of 5 minutes and the mean time was considered.

\section{Visual analog scale (VAS) - for sedation ${ }^{10}$}

Subject was instructed to mark intensity of sleep on VAS for sedation of $100 \mathrm{~mm}$, by a vertical line [0 indicates not sleepy and 100 indicates extremely sleepy] distance on VAS is recorded as $\mathrm{mm}$.

\section{Statistical analysis}

Data was expressed as mean \pm SD. Paired t- test was performed within groups and unpaired t test was done for between groups analysis. A p-value of less than 0.05 was considered to be statistically significant.

\section{RESULTS}

Among twelve subjects enrolled in study, eight were males and four were females. The mean age of the population was $23.1( \pm 1.5)$ years. The mean body mass index of the population was $22.75( \pm 1.2) \mathrm{Kg} / \mathrm{m}^{2}$.

With Agomelatine, subjects showed statistical significant difference in choice reaction time $(\mathrm{p} \leq 0.01)$ and improved performance in critical flicker fusion test $(p \leq 0.05)$ when compared to the baseline. Psychomotor functions did not show significant change in digit letter substitution test, six letter cancellation test, card sorting test, before and after administration of Agomelatine. There was a significant difference of mean score of VAS values for sedation with $\mathrm{p}$ value $(<0.0001)$ before and after administration of Agomelatine. Figure 1 shows the mean change of psychomotor functions before and after administration of Agomelatine.

There was no statistical significant difference seen with Choice reaction time, critical flicker fusion test, digit letter substation test, six letter cancellation test and card sorting test, before and after administration of placebo. Figure 2 depicts the mean change of psychomotor functions pre and post placebo administration.

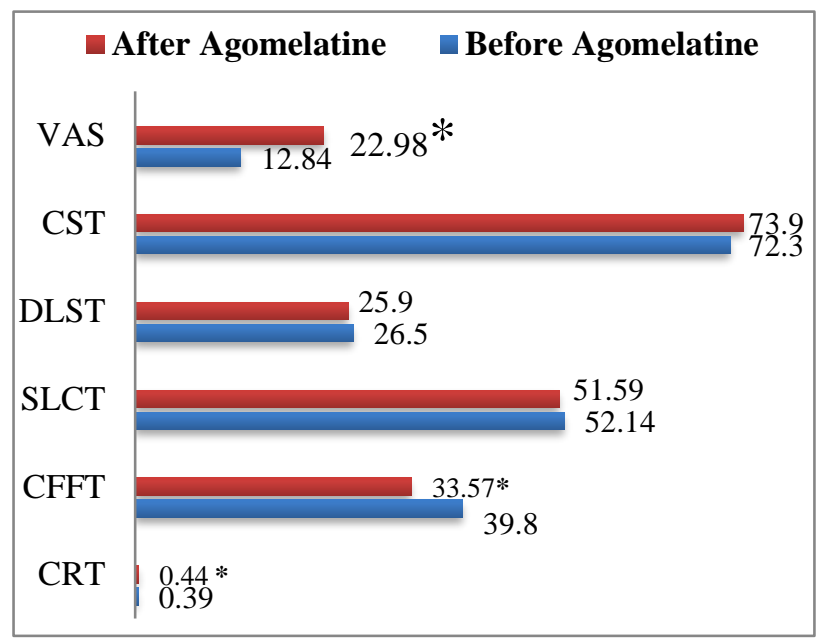

Figure 1: Effect of agomelatine 25mg on psychomotor function tests.

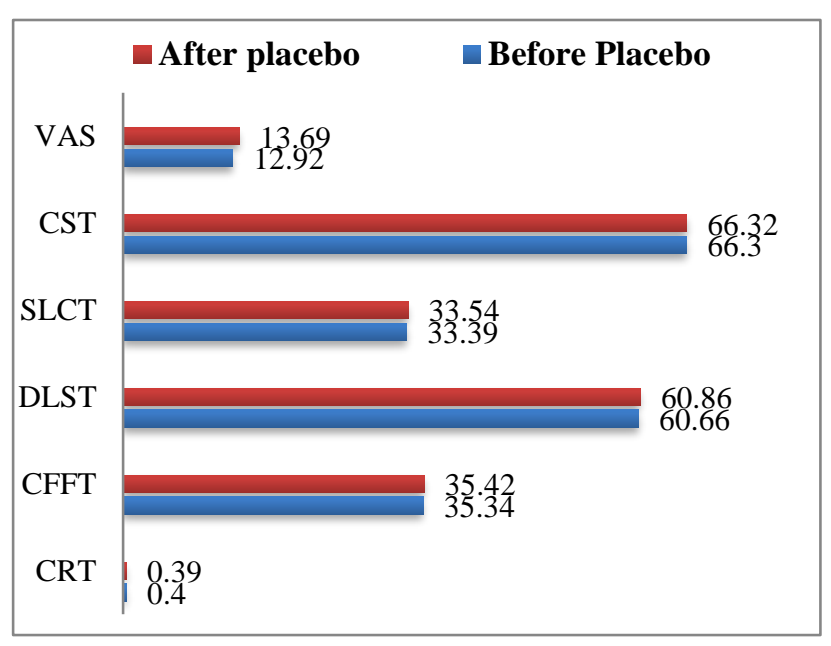

Figure 2: Effect of placebo on psychomotor function tests.

There was significant decrease in mean percentage time $(\mathrm{p} \leq 0.01)$ in choice reaction time test in Agomelatine group $(20.09 \pm 9.47 \%)$ compared to placebo $(10.48 \pm 3.68)$. There was improved performance in critical flicker fusion test $(\mathrm{p} \leq 0.01)$, when mean percentage change in performance in Agomelatine group $(6.2 \pm 2.1)$ was compared to placebo $(9.11 \pm 2.99)$. There was no significant change in mean percentage of performance in digit letter substitution test and six letter cancellation test in Agomelatine group compared to placebo. There was no significant decrease in mean percentage time to sort cards in card sorting test in Agomelatine group compared to the placebo group. Agomlatine cause significant sedation on visual analog scale in Agomealtine group compared to placebo group. Table 1 shows the comparisons of the mean performance between Agomelatine and placebo group. 
Table 1: Percentage change in performance in Agomelatine and placebo groups compared to baseline values.

\begin{tabular}{|c|c|c|c|}
\hline $\begin{array}{l}\text { Psychomotor } \\
\text { function } \\
\text { tests }\end{array}$ & $\begin{array}{l}\text { Agomelatine } \\
\text { (\% change) }\end{array}$ & $\begin{array}{l}\text { Placebo } \\
\text { (\% change) }\end{array}$ & $\begin{array}{l}\mathrm{p} \\
\text { value }\end{array}$ \\
\hline $\begin{array}{l}\text { Choice } \\
\text { reaction test }\end{array}$ & $16.39 \pm 8.54$ & $10.48 \pm 3.68$ & $0.002 *$ \\
\hline $\begin{array}{l}\text { Critical } \\
\text { Flicker } \\
\text { Fusion test }\end{array}$ & $6.2 \pm 2.1$ & $9.11 \pm 2.99$ & $0.01 *$ \\
\hline $\begin{array}{l}\text { Digit letter } \\
\text { substitution } \\
\text { test }\end{array}$ & $11.3 \pm 7.45$ & $9.1 \pm 5.21$ & $0.84(\mathrm{NS})$ \\
\hline $\begin{array}{l}\text { Six letter } \\
\text { cancellation } \\
\text { test }\end{array}$ & $8.82 \pm 6.38$ & $12.08 \pm 8.07$ & $0.22(\mathrm{NS})$ \\
\hline $\begin{array}{l}\text { Card sorting } \\
\text { test }\end{array}$ & $10.35 \pm 5.26$ & $2.61 \pm 1.41$ & $0.14(\mathrm{NS})$ \\
\hline
\end{tabular}

$\mathrm{p}$ value $\leq 0.01^{*}, \mathrm{NS}-$ not significant

All the subjects tolerated study medications except two subjects who reported with headache and one patient complained of dizziness which resolved spontaneously. No subject discontinued the study due to serious adverse events.

\section{DISCUSSION}

All psychoactive drugs need to be screened for their effect on the Central Nervous System. These tests should be highly sensitive and reliable to pick the change in CNS function. Tests that measure CNS function include 1. Tests for attention evaluation 2. Tests for psychomotor performance 3 . Tests for memory 4 .Tests for cognitive function.

Psychomotor retardation is a central feature of depression that can have clinical and therapeutic implications. This includes both motor and cognitive impairments, affecting speech, motility, and ideation. These symptoms may severely impact patient's psychosocial functioning and are closely linked with severity of depression. ${ }^{11}$

Cognitive function is brain's ability to acquire process, integrate, store and retrieve information. ${ }^{12}$ It is known that cognition and psychomotor function becomes impaired with age, due to depression and as a result of treatment with antidepressant drugs. Deficits in memory function, executive function, attention, and psychomotor speed also occur in patients with Major Depressive Disorder (MDD).

Agomelatine, an antidepressant by virtue of its agonistic effect on melatonin receptors, was studied in healthy human volunteers for its effect on attention, cognition and information processing using a battery of psychomotor function tests. Since depressed mood is a pivotal criterion for the diagnosis of a depressive episode, drug-placebo separation on this item is given particular attention in evaluating antidepressant efficacy. Ramelteon, a prototype of this class of antidepressants was shown to impair memory, cognitive and psychomotor functioning. ${ }^{13}$

Agomelatine improves the quality of sleep without day time sedation. ${ }^{14,15}$ Sedating drugs generally impair both controlled and automatic information processing simultaneously by reducing the brain's level of arousal that energizes both. Controlled information processing is usually a conscious process involving the effortful extraction of information to:

- $\quad$ Determine meaning

- Decision making

- Response action

- $\quad$ Retention of the whole process in memory

Deficits in controlled processing have been attributed to impaired central executive functioning. Automatic information processing occurs subconsciously without perceived effort after brain has established a particular sensory input- motor output relationship, usually after extensive practice. Once a particular feedback loop is established, the process ceases to add information to memory.

Choice reaction time is an index of alertness and motor speed. The results obtained in CRT, indicate that Agomelatine impairs alertness and motor speed. Critical flicker fusion test measures information processing in CNS and Agomelatine this study has shown to improve information processing. This potential of Agomelatine to improve psychomotor performance makes it safe and promising novel antidepressant.

Digit letter substitution test measures psychomotor speed and visual motor co-ordination. Performance in DLST requires visual performance, spatial decision making and motor skills, which are not significantly impaired as shown by the results in the present study. The results were also comparable with Ramelteon, another melatonergic agoinst when DLST was performed. ${ }^{16,17}$ Six letter cancellation test measures attention and perceptual processing of sensory information, which is parietal lobe function. In the present study, it is shown that Agomelatine has no effect on sensory perception. The card sorting test evaluates the overall CNS executive function, which is frontal lobe function. The overall executive function of CNS is not impaired significantly, with the use of Agomelatine, as seen in the present study.

Visual analog scale for sedation rates alertness, calmness, drowsiness and contentment subjectively. In a previous study, it was shown that Agomelatine was associated with significant reduction in sleep latency from week 2 and improvement in sleep efficiency. ${ }^{18}$ Its sedative action is particularly useful in sleep related disturbances of depressed patients. ${ }^{19,20}$ 
Our study had few limitations. Firstly, the acute effect on psychomotor performance in healthy subjects is a validated method for evaluating a drug which causes sedation. ${ }^{20}$ However, it should be noted that the results of our acute experiment may not be directly applicable to patients, who are treated for a chronic period of time. Although Agomelatine has shown improvement in overall integrative and sensory processing mechanism of psychomotor performance, it requires similar evaluation in depressed patients to establish its beneficial effect on psychomotor performance. In our study, 12 healthy volunteers were taken up. Studies with larger sample size are recommended for better yield of results.

\section{CONCLUSION}

In our study, CFFT is decreased with Agomelatine which indicates on improvement in information processing in CNS. Also, there is no significant effect on psychomotor performance with Agomelatine. No significant adverse effects were reported. The drug can thus be safely administered to depressed patients.

Funding: No funding sources

Conflict of interest: None declared

Ethical approval: The study was approved by the Institutional Ethics Committee

\section{REFERENCES}

1. Katzung BG. Basic \& Clinical Pharmacology 11ed.New York: Lange Medical Publications; 2009:509.

2. Srinivasan VR, Zakaria R, Othman Z, Edward CL, Dario AC. Agomelatine in depressive disorders: its novel mechanisms of action. The $\mathrm{J}$ of Neuropsychiatry and Clinical Sciences. 2012;24(3):290-308.

3. Kennedy HS, Cyriac A. A dimensional approach to measuring antidepressant response: implications for Agomelatine. Psychology. 2013;3(10):864-9.

4. Girish MB, Bhuvana K, Nagesh Raju G, Sarala N. A novel atypical antidepressant drug: A review. Int J Pharm Biomed Res. 2010;1(3):113-6.

5. Hindmarch I. Psychomotor function and psychoactive drugs. Br J Clin Pharmac. 1980;10:189-209.

6. Kasper S, Hajak G, Wulff K, Hoogendijk WJG, Montejo AL, Smeraldi E, et al. Efficacy of Novel antidepressant Agomelatine on circadian rest activity cycle and depressive and anxiety symptoms in patients with Major Depressive Disorder: A randomized, double blind comparison with Sertraline. J Clin Psychiatry. 2010;71(2):109-20.

7. Stone BM. Pencil and paper tests: sensitivity to psychotropic drugs. Br J Clin Pharmac. 1984;18:155205.
8. Turner P. Critical flicker frequency and centrally acting drugs. Br J Ophthal. 1968;52:245-50.

9. Balaram P. Effect of kapalbhati on performance of six letter cancellation test and digit letter substitution task in adults. Int J Yoga. 2013 Jul-Dec;6(2):128-30.

10. Aitken RCB. Measurement of feelings using visual analogue scales. Proc Roy Soc Med. 1969;62:989-93.

11. Nelson JC, Charney DS. The symptoms of major depressive illness. The American J of Psychiatry. 1998;138(1):1-13.

12. Roger ML, Hanlon FJ. Cognitive and Psychomotor Effects of Antidepressants with Emphasis on Selective Serotonin Reuptake Inhibitors and the Depressed Elderly Patient. German J Psychiatry. 1999;2(1):5180.

13. Mets AJ, Deventer KR, Olivier B, Verster JC. NextDay Effects of Ramelteon (8mg), Zopiclone (7.5mg), and Placebo on Highway Driving Performance, Memory Functioning, Psychomotor Performance, and Mood in Healthy Adult Subjects.

14. Michele F, Davide P, Salvatore C, Giulio P. A Systematic, Updated Review on the Antidepressant Agomelatine Focusing on its Melatonergic Modulation. Current Neuropharmacology. 2010;8:287-304.

15. Srinivasan V, Pandi-Perumal SR, Trakht I, Spence DW, Hardeland R, Poeggeler B. Pathophysiology of depression: role of sleep and the melatonergic system Psychiatry Res. 2009 Feb 28;165(3):201-14.

16. Mets AJ, Deventer KR, Olivier B, Verster JC. Critical appraisal of ramelteon in the treatment of insomnia. Nat Sci Sleep. 2010;2:257-66.

17. Gary Z, Milton E. Evaluation of the Efficacy and Safety of Ramelteon in Subjects with Chronic Insomnia. J Clin Sleep Med. 2007 Aug 15;3(5):495504.

18. Bertaina-Anglade V, Drieu-La-Rochelle C, Mocaër E, Seguin L. Memory facilitating effects of agomelatine in the novel object recognition memory paradigm in the rat. Pharmacol. Biochem. Behav. 2011;98:511-7.

19. Sarfaraz AK, Mukesh CM, Anand K, Avinash T. Acute Effects of Citalopram and Imipramine on Psychomotor Performance in Healthy Volunteers: A Comparative Study. J Pharm Sci \& Res. 2011;3(6):1269-75.

20. Jhanjee A, Bhatia MS, Shruthi S, Kumar P. Agomelatine: A new antidepressant with a novel mechanism of action. Delhi Psychiatry J. April 2010;13(1):170-8.

Cite this article as: Sirisha G, Shovan P. Effect of agomelatine on psychomotor function tests in healthy human volunteers. Int J Basic Clin Pharmacol 2018;7:109-13. 